\title{
In Situ Imaging of Layer-by-Layer Sublimation of Suspended Graphene
}

\author{
Jian Yu Huang ${ }^{1}(\varangle)$, Liang Qi ${ }^{2}$, and $\mathrm{Ju}^{\mathrm{Li}^{2}}$ \\ ${ }^{1}$ Center for Integrated Nanotechnologies (CINT), Sandia National Laboratories, Albuquerque, New Mexico 87185, USA \\ ${ }^{2}$ Department of Materials Science and Engineering, University of Pennsylvania, Philadelphia, Pennsylvania 19104, USA \\ Received: 5 October 2009 / Revised: 20 November 2009 / Accepted: 21 November 2009 \\ (C) The Author(s) 2010. This article is published with open access at Springerlink.com
}

\begin{abstract}
An individual suspended graphene sheet was connected to a scanning tunneling microscopy probe inside a transmission electron microscope, and Joule heated to high temperatures. At high temperatures and under electron beam irradiation, the few-layer graphene sheets were removed layer-by-layer in the viewing area until a monolayer graphene was formed. The layer-by-layer peeling was initiated at vacancies in individual graphene layers. The vacancies expanded to form nanometer-sized holes, which then grew along the perimeter and propagated to both the top and bottom layers of a bilayer graphene joined by a bilayer edge. The layer-by-layer peeling was induced by atom sublimation caused by Joule heating and facilitated by atom displacement caused by high-energy electron irradiation, and may be harnessed to control the layer thickness of graphene for device applications.
\end{abstract}

\section{KEYWORDS}

Graphene, sublimation, in situ electron microscopy, vacancy hole, bilayer edge

\section{Introduction}

Graphene is a two-dimensional (2-D) crystal that exhibits intriguing electronic [1, 2], mechanical [3], and thermal properties [4]. Graphene is also an interesting material for electron microscopy [5-11]. By using a new generation of transmission electron microscopes (TEMs) which are known as aberration corrected TEMs (AC-TEMs), individual carbon atoms in a graphene layer can be imaged [5-9], which provides an unprecedented opportunity to probe the physical behavior of individual defects such as a vacancy in graphene, and also offers the capability to probe truly at an atomic scale the edge reconstructions in graphene. Most significantly, these results can be directly compared with theoretical modeling to test the validity of a variety of modern theories [12,13]. In this context, graphene is a test bed for fundamental theory regarding how matter is assembled or disassembled at an atomic level. On another front, graphene also offers a unique opportunity for in situ electron microscopy [10,11]. By Joule-heating graphene layers to high temperatures inside a highresolution TEM (HRTEM), the edge reconstructions or evolution can be directly imaged in real time $[10,11]$. The results provide important insights into how to shape the graphene edge or pattern its structure by Joule heating and electron irradiation in order to fabricate materials for specific device applications.

Address correspondence to jhuang@sandia.gov or jyhuang8@yahoo.com 
Despite its potential important applications, controlling the layer thickness of graphene still remains a challenge. The thickness of as-synthesized graphene ranges from a single to tens of layers [1]. It has been shown that the layer thickness can significantly affect the electronic properties of graphene [14]. Here we report a technique, based on Joule heating, to peel off the multilayer suspended graphene sheets layer-bylayer locally until only single-layer graphene remains The results offer a new approach to engineering the graphene layer thickness.

\section{Experimental}

The experiments were conducted in a TEM equipped with a Nanofactory scanning tunneling microscopy (STM) probe $[11,15]$. TEM experiments were conducted in a Tecnai $G^{2}$ TF30 S-Twin microscope operated at $300 \mathrm{kV}$ with a point-to-point resolution of
$0.2 \mathrm{~nm}$. The TEM samples were prepared by the following procedure. First, a block of highly orientated pyrolytic graphite (HOPG) was mounted on a glass slide with double-sided adhesive tape (Fig. 1(a)). Second, the HOPG was thinned down to transparent under an optical microscope using the Scotch tape method. Third, a half TEM grid with conductive epoxy painted on the grid bars was glued to a transparent graphene sheet under the optical microscope. After the epoxy was cured, the half TEM grid was lifted up by tweezers and glued to a gold rod. Individual graphene sheets with layer thickness ranging from a few to tens of layers were produced in this way (Fig. 1(b)). Finally, the whole assembly was inserted into a TEM-STM holder (Fig. 1(c)) [11, 15]. The STM probe inside the TEM sample holder can be manipulated to approach and contact individual graphene sheets (Figs. 1(b) and 1(c)). Once a contact between the STM probe and a graphene sheet was

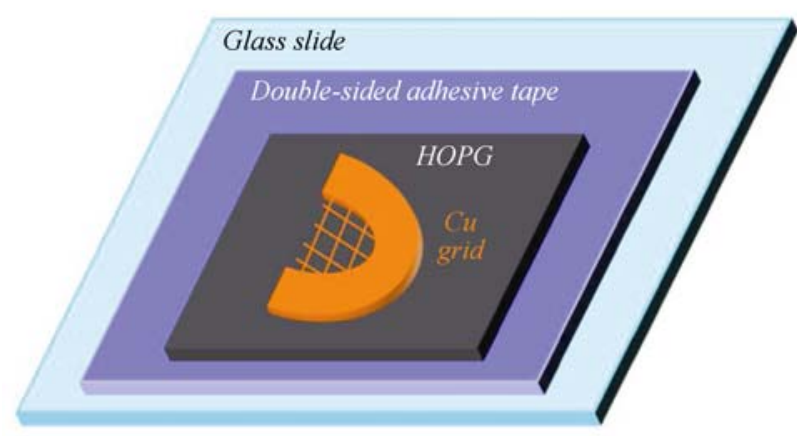

(a)

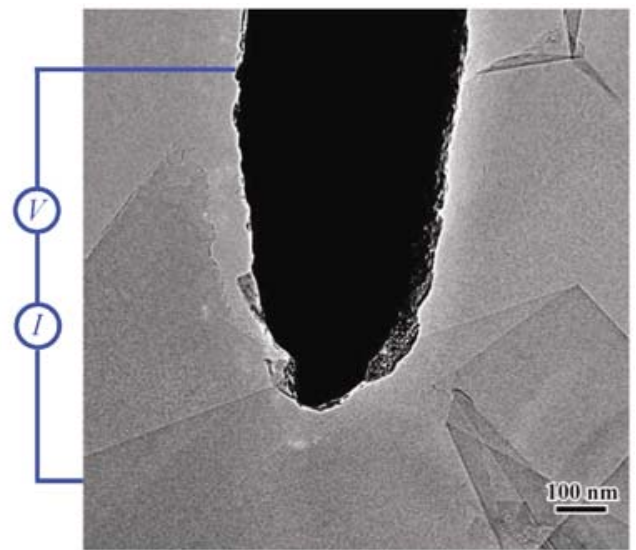

(b)

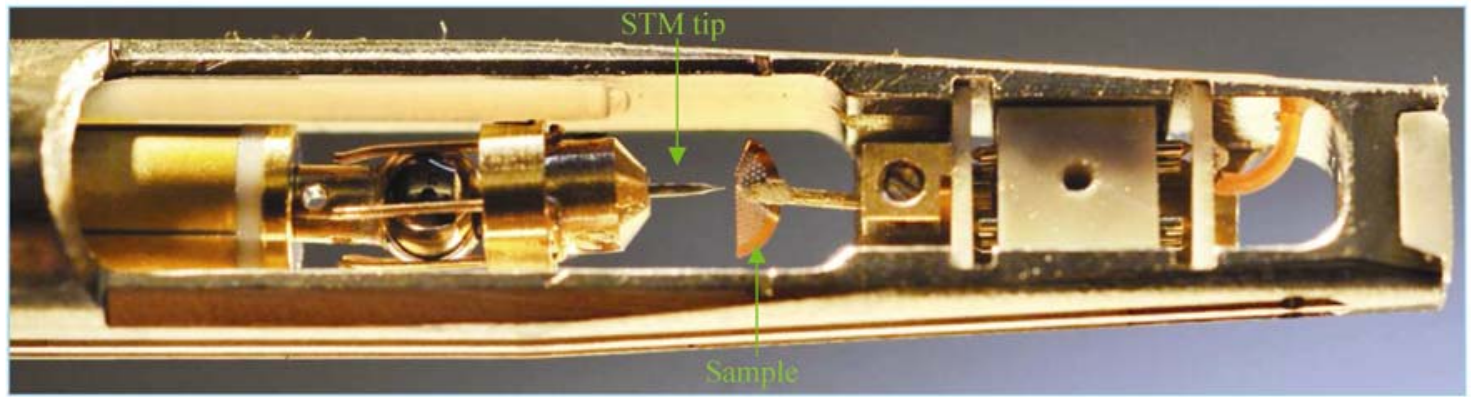

(c)

Figure 1 (a) A schematic drawing showing the TEM sample preparation procedure. A block of HOPG was attached to a glass slide by two-sided adhesive tape. The HOPG was thinned down to one to a few layers by repeated Scotch tape peeling. A half $\mathrm{Cu}$ grid with conducting glue on the grid bars was glued on a thin graphene sheet. After the glue was cured, the $\mathrm{Cu}$ grid was lifted off and attached to an Au rod as shown in (c). (b) A TEM image and a schematic drawing showing the experimental setup. An individual graphene sheet was connected to an STM probe and then Joule-heated to high temperatures. (c) The TEM-STM holder 
established, a high electrical current was then passed through the graphene to Joule-heating the graphene to its sublimation temperature. At high temperatures and under electron beam irradiation, layer-by-layer peeling of graphene occurred.

\section{Results and discussion}

The sublimation process generally followed the steps shown schematically in Fig. 2. At high temperatures, the neighboring graphene edges became joined together by a nanoarch or a fractional carbon nanotube to form bilayer edges (BLEs) $[7,11]$. These BLEs are much more stable than a monolayer edge (MLE) because there are no dangling bonds in a BLE. Once two BLEs approached each other, they reconstructed to form nested BLEs (Fig. 2(a)), which are similar to a fractional double-walled carbon nanotube (DWCNT) [16]. The spacing between two nested BLEs is similar to that between the two walls in a DWCNT. Similarly, once multiple BLEs approached very closely, they formed multiple nested BLEs similar to a fractional multi-walled carbon nanotube (MWCNT). Vacancies were created in individual graphene layers by Joule heating and facilitated by electron beam irradiation. The vacancies grew to form an elliptical hole (Fig. 2(a)), which then expanded along its perimeter until it encountered a BLE. The holes either followed the BLE and propagated to the bottom layer of the bilayer graphene (Figs. 2(b) and 2(c)) or migrated to the inner BLE (not shown in the figure). A similar process was repeated with the inner bilayer graphene until a monolayer graphene was formed.

Figure 3 (Movie S-1 in the Electronic Supplementary Material (ESM)) shows the peeling process of two-nested bilayer graphene (a four-layer stack, see Figs. 3(a) and 3(b)). As shown in our previous study, determination of graphene layer thickness by counting lattice fringes of the graphene edge is problematic [11]. A single fringe can represent either an MLE or a BLE. A BLE usually shows much darker contrast compared to an MLE under the same imaging conditions, while MLEs show a faint contrast. We conducted image simulations on a BLE and an MLE under the same image conditions, which showed that the contrast difference between the two types of edges is about $30 \%$ (Figs. S-1(a) and S-1(b) in the ESM). We measured the intensity profiles of the segments of the MLE and the BLE shown in Fig. 3(c), and the intensity difference between the two edges was $26 \%$ (Figs. S- 1 (c) and S- 1 (d) in the ESM), which is close to the simulation results. Measurements conducted on more experimental images of the BLEs and the MLEs showed that their intensity difference ranges from $15 \%$ to over $50 \%$. In addition to the BLEs formed by the reconstruction, an MLE can also roll up to form a BLE (Fig. S-2, Movie S-2 in the ESM). Figure S-2(a) in the ESM shows an MLE, which first rolled up to form a BLE (Fig. S-2(b)), and subsequently unwrapped thus regenerating an MLE (Fig. S-2(c)). Once the BLE was formed (Fig. S-2(b)), its contrast became much stronger than that of the MLE (compare

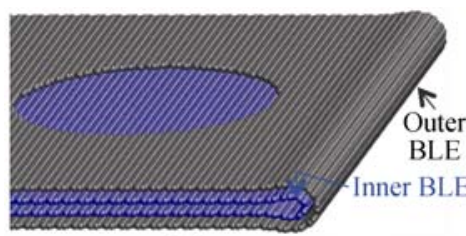

(a)

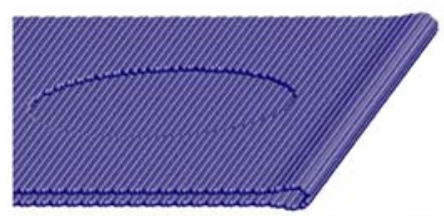

(d)

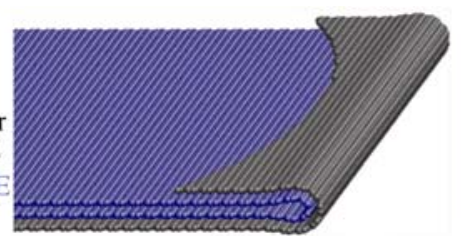

(b)

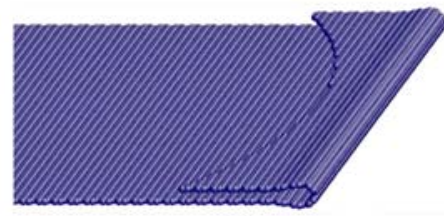

(e)

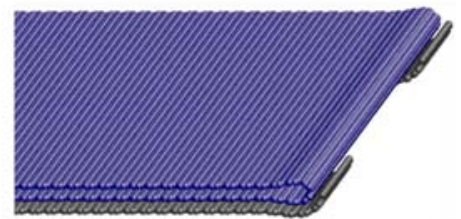

(c)

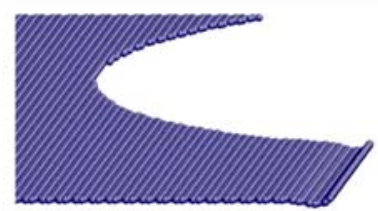

(f)

Figure 2 Schematic drawings showing a layer-by-layer sublimation of two-nested bilayer graphenes, which are joined by a BLE. The elliptical hole in (a) indicates a vacancy hole 
Figs. S-2(a) and S-2(b)). The wrapping and unwrapping processes can be more clearly seen in Movie S-2 in the ESM.

In Fig. 3(c), there are two dark fringes in the graphene edge, but this graphene sheet actually has four layers, and each fringe represents a BLE as explained further in what follows. In Fig. 3(c), the fourth layer (the blue layer in Figs. 3(a) and 3(b)) was almost burned away (its edge is highlighted in bluedotted lines), then the third layer (the red layer in Figs. 3(a) and 3(b)) started to sublime (its edge is marked by red-dotted lines, Figs. 3(d)-3(f)) until it was almost completely sublimed in the area of view (Fig. 3(g)). Note that layer 4 (the blue layer in Figs. 3(a) and 3(b)) and layer 3 (the red layer in Figs. 3(a) and 3(b)) were the top and bottom layers, respectively, of a bilayer graphene that was joined by a BLE (highlighted by the overlapping blue- and red-dotted lines in Figs. 3(c)-3(f)). The sublimation occurred simultane- ously at the top (the blue layer in Figs. 3(a) and 3(b)) and bottom (the red layer in Figs. 3(a) and 3(b)) layers. Counterintuitively, in this case the sublimation occurred first at the inner bilayer graphene rather than at the outer bilayer. A similar phenomenon was observed in MWCNTs, in which sublimation occurred at the inner walls rather than at the surface wall [17]. The sublimation continued on the remaining bilayer graphene (the black and green layers in Figs. 3(a) and 3(b) (the edges are denoted in black- and green-dotted lines in Figs. 3(g)-3(j)) until a monolayer graphene was produced (Fig. 3(j)). The MLE was not straight but rather wavy, in contrast to a BLE which is usually straight and faceted on a zigzag or armchair inclination. The reason for such different edge configurations is reported elsewhere [18]. The sublimation also sometimes started from the external surface bilayer. Once a vacancy or vacancy clusters were formed, the sublimation then continued along the vacancy

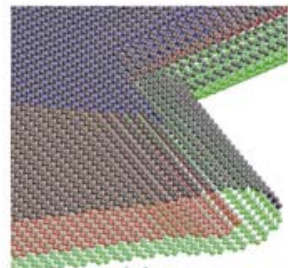

(a)
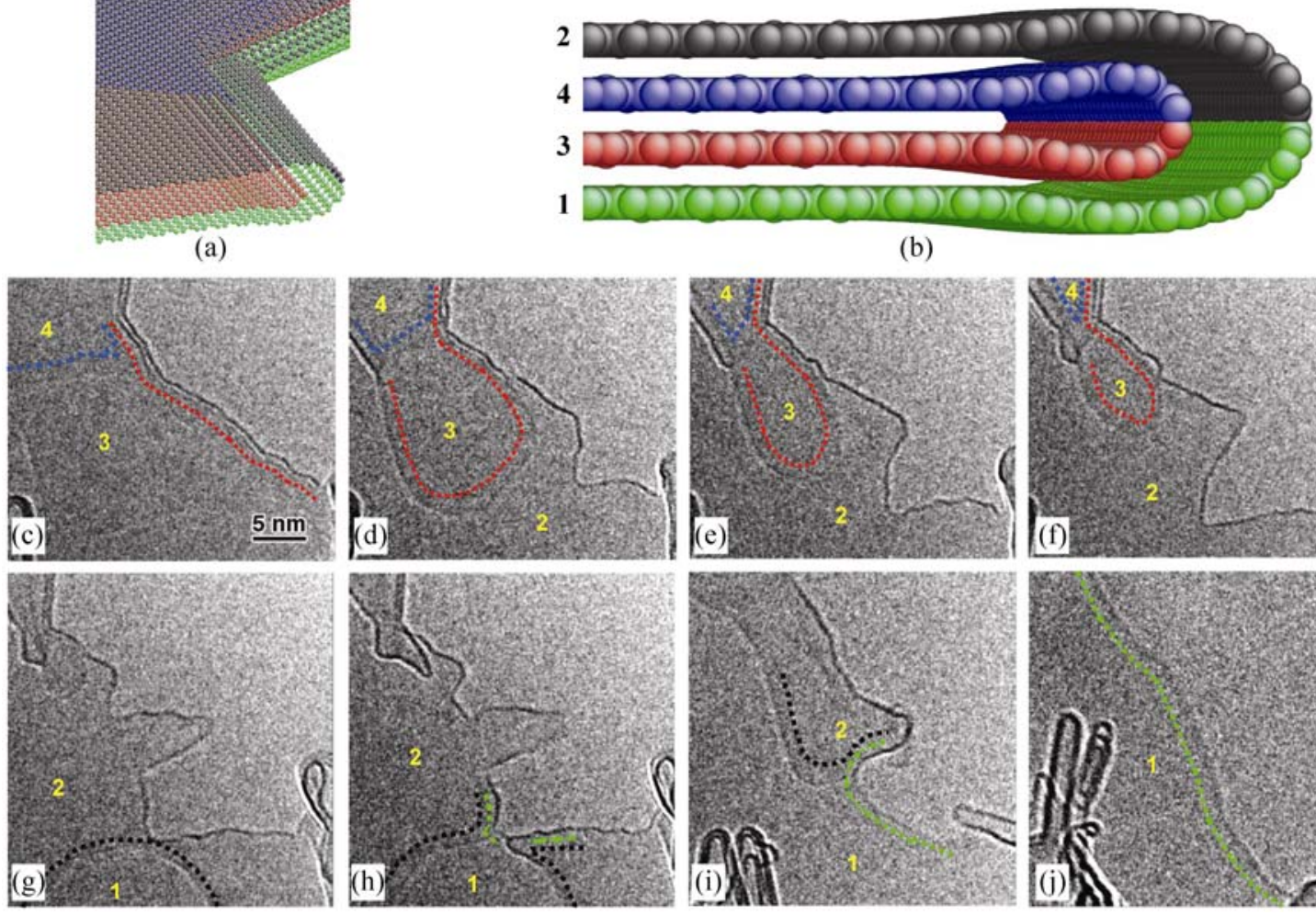

(b)

Figure 3 (a) and (b) are plan- and side-views of the initial structure of two nested BLEs, which were then sublimed layer-by-layer by Joule heating as shown in (c) to (j) and also in Movie S-1. The dotted lines in (c) to (j) indicate the layer edges, where the color of one line indicates that the edge is located on the layer with the same color shown in (a) and (b). The numbers in (c) to (j) indicate the layer numbers that correspond to the layer numbers indicated in (b). In (j) a single layer graphene was produced. The layer sublimation sequence is: blue $\rightarrow$ red $\rightarrow$ black $\rightarrow$ green, or $4 \rightarrow 3 \rightarrow 2 \rightarrow 1$. The bias voltage applied to the graphene was $2.5 \mathrm{~V}$ 
perimeter. This can be understood by the fact that removing an atom from a vacancy edge requires much less energy $(\sim 5 \mathrm{eV})$ than that from a perfect lattice site $(\sim 30 \mathrm{eV})[6,19]$. The carbon atom edge diffusion along an MLE has previously been observed to be fast $[6,20]$. So as long as the MLEs on inner layers percolate and form a connection to the outside, edge diffusion can be an effective mass transport channel that feeds the sublimation to the gas phase. The process is similar to the mass transport along a dislocation line in an MWCNT [17].

Figure 4 (Movie S-3 in the ESM) shows the layerby-layer removal process in a two-nested bilayer and a sandwiched monolayer graphene (Figs. 4(a) and 4(b)). An elliptical vacancy hole emerged on the top layer (the black layer in Fig. 4(b) - the hole edge is marked by black dotted lines in Fig. 4(c)), then expanded (Fig. 4(d)), and hit the outer BLE (marked by a left-pointing white arrow in Fig. 4(d)), which then migrated to a neighboring layer (the blue layer in Fig. 4(b) - the hole is illustrated by blue dotted lines in Figs. 4(e) and 4(f)) and induced fast sublimation of this graphene layer (Figs. 4(f) and 4(g)). Meanwhile, a second elliptical vacancy hole emerged on the red layer (Figs. 4(b) and 4(f) - the hole edge is marked by red dotted elliptical lines in Figs. 4(f) and 4(g)), which then grew and migrated upwards (Fig. 4(g)). Thus, the sublimation occurred simultaneously on the top (the blue layer in Fig. 4(b)) and bottom (the red layer in Fig. 4(b)) layers of the same bilayer graphene joined by the inner BLE until almost the whole inner bilayer was etched away (Figs. 4(g)-4(i)). In the next step, a third hole was formed on the monolayer graphene (the green layer in Fig. 4(b)) sandwiched inside the BLEs (Figs. 4(j) and 4(k), shown by green dotted lines), and grew until the entire layer was sublimed. During sublimation, we can also see new BLEs were formed from two conjoined MLEs (highlighted by the overlapping red- and green-dotted lines in Figs. 4(j) and $4(\mathrm{k}))$. Then, only two outer graphene layers connected to the outer BLE were left, but another void emerged on one of them and grew (Fig. 4(1), yellow dotted lines), so that the layer-by-layer etching continued until only a single layer was left. We should point out that here we assume the sublimation started from the top layer (for convenience of description), but the sublimation could also initiate from the bottom layer due to preferred electron beam sputtering of the bottom layer. Nevertheless, the layer-by-layer sublimation process is essentially similar, regardless of where the holes were first formed.

The layer-by-layer etching is caused by atom sublimation induced by Joule heating and high-energy electron beam sputtering. The temperature in the Jouleheated graphene could not be determined precisely, but the sublimation temperature of graphite in vacuum is about $2500 \mathrm{~K}$ [20]. Since the sublimation was assisted by electron beam sputtering, the sublimation temperature in our experiments could be lower than $2500 \mathrm{~K}$. Without Joule heating, layer-by-layer peeling did not occur and, instead, the graphene layers quickly became perforated amorphous structures due to irradiation damage. This was particularly so when only a few layers were present. Figure 5(a) is a typical HRTEM image of a graphene layer with no Joule heating. One can see the graphene is highly disordered and perforated. After passing a high current with a current density of about $10^{8} \mathrm{~A} / \mathrm{cm}^{2}$, clean graphene with well defined edges appears (Fig. 5(b)). It was reported recently that layer-by-layer peeling occurred in graphene only on electron beam irradiation at $80 \mathrm{kV}$ [6]. In our experiments, the TEM was operated at $300 \mathrm{kV}$, and the knock-on threshold voltage for carbon atoms is $\sim 80 \mathrm{kV}$. The maximum energy transfer from an $80-\mathrm{keV}$ electron to a carbon atom is $15.8 \mathrm{eV}$ $[6,19]$, while the displacement threshold for a carbon atom in a perfect honeycomb lattice is $\sim 30 \mathrm{eV}[6,19]$. However, the displacement energy for a carbon atom near a vacancy is much lower, less than $5 \mathrm{eV}[5,6]$, so the sputtering and sublimation will preferentially occur along the exposed edge or the void edge, which are both MLEs. The void in a single-layer graphene often starts with a single vacancy, which then expands rapidly along the edge until the one layer is eliminated. In our experiments, because both heat and electron beam sputtering contribute to the atom removal, and furthermore the electron beam energy ( $300 \mathrm{keV})$ is much higher than $80 \mathrm{keV}$, the atom removal speed is much faster than in all previously reported experiments. 

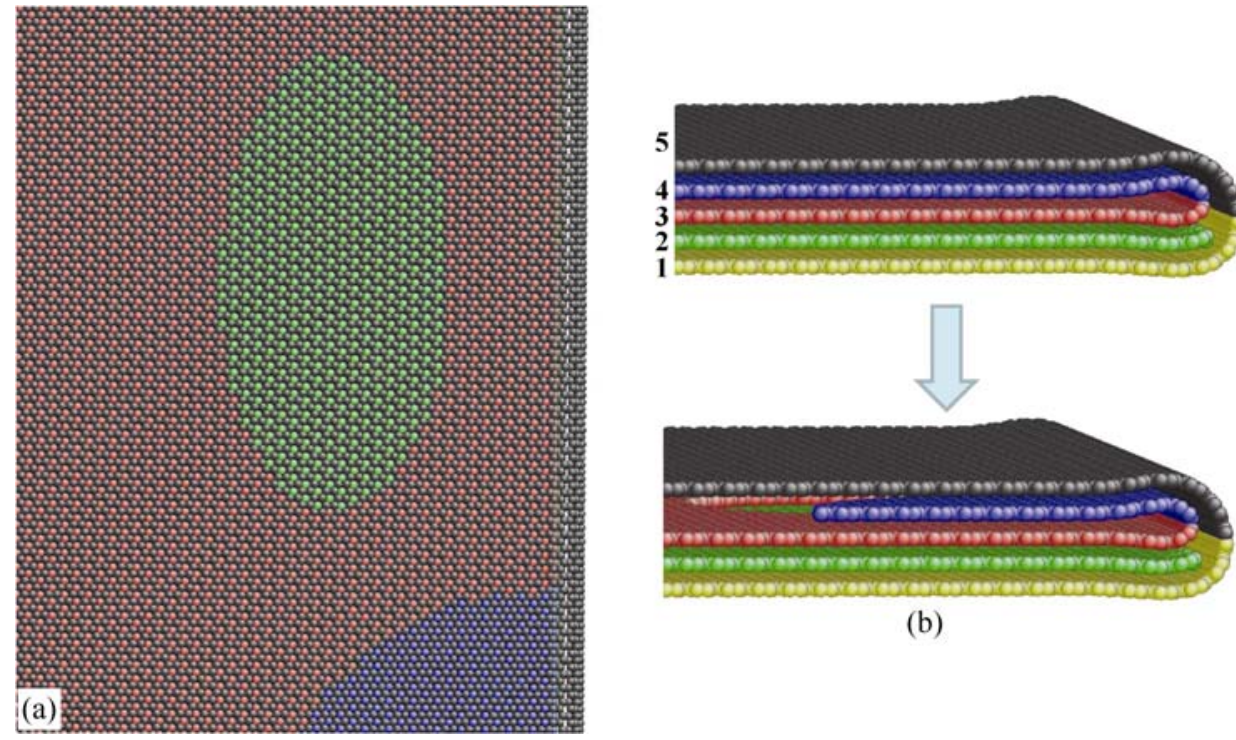

(b)
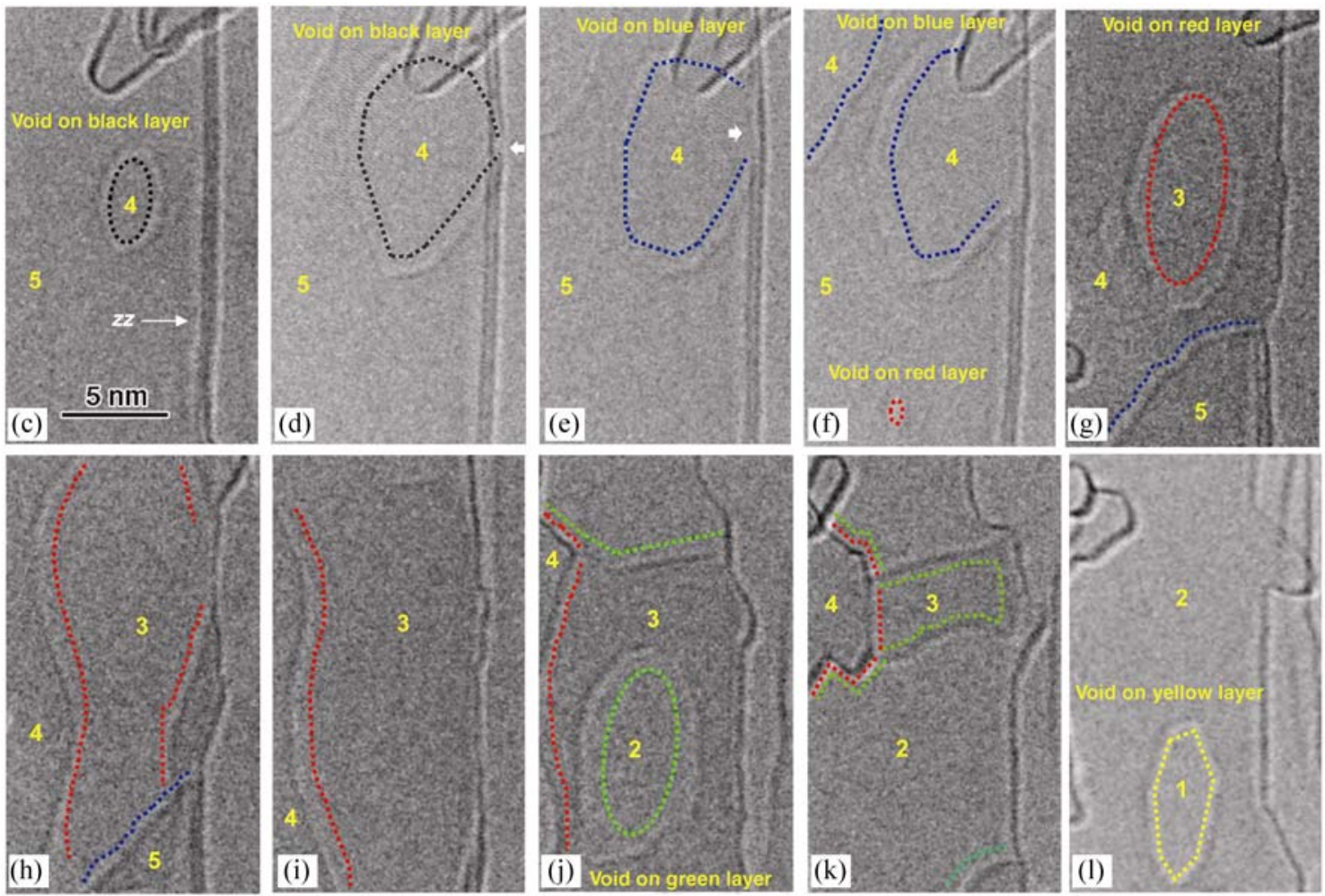

Figure 4 (a) and (b) are plan- and side-views of the initial structure of two nested BLEs with an MLE, which were then sublimed layer-by-layer by Joule heating as shown in (c) to (1) and also in Movie S-3. Dotted lines in (c) to (1) indicate the vacancy hole or graphene edges, where the color of one line indicates that the edge is located on the layer with the same color shown in (a) and (b). The numbers in (c) to (j) indicate the layer numbers that correspond to the layer numbers indicated in (b). The locations of the holes are also indicated in (c)-(1). The bias voltage applied to the graphene was $2.5 \mathrm{~V}$ 

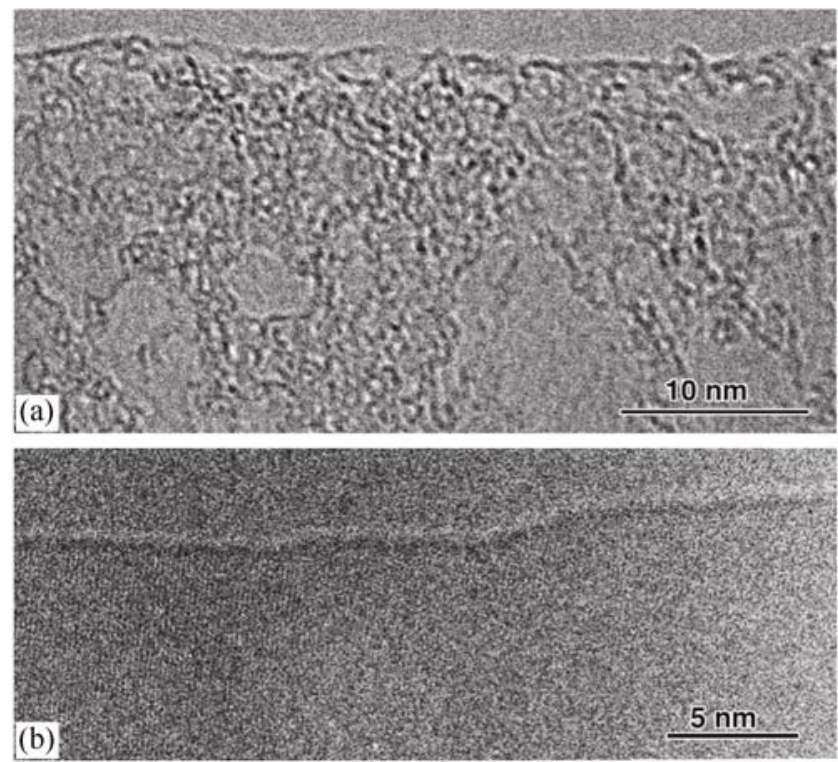

Figure 5 (a) An HRTEM image of a thin graphene without Joule heating, showing the disordered and perforated structure. (b) An HRTEM image of a graphene exposed to Joule heating showing a clean surface and well-developed edge. The current density required to achieve such crystallinity is about $10^{8} \mathrm{~A} / \mathrm{cm}^{2}$

\section{Conclusions}

Although a layer-by-layer peeling phenomenon has been observed with carbon nanotubes subjected to only Joule heating or a combination of Joule heating and electron beam irradiation [15, 21, 22], it is difficult to image the peeling mechanism in nanotubes due to their curved tubular structure. Graphene offers the advantage that it is flat, and vacancy defects can be clearly imaged. The sublimation observed for graphene started with the formation of vacancy holes, which then grew until the entire layer was eliminated. The vacancy hole activity in graphene is similar to that in a carbon nanotube [23, 24]. The results thus also shed light on the mechanism of breakdown of carbon nanotubes. This is apparently a defect-, and particularly vacancy-, mediated process. We believe that layer-by-layer peeling should occur by Joule heating alone for graphene with appropriate graphene geometry and contacts-such as a graphene ribbon with contacts at its two ends-which may provide an important technique for tailoring the graphene layer thickness with an atomic precision for device applications.

\section{Acknowledgements}

This work was performed, in part, at the Center for Integrated Nanotechnologies, the U. S. Department of Energy, Office of Basic Energy Sciences user facility. Sandia National Laboratories is a multi-program laboratory operated by Sandia Corporation, a Lockheed-Martin Company, for the U. S. Department of Energy under Contract No. DE-AC04-94AL85000. JL would like to acknowledge support by Honda Research Institute USA, NSF CMMI-0728069, AFOSR, and ONR N00014-05-1-0504. J. Y. H. would like to thank Dr. Ping Lu at Sandia National Laboratories for conducting the HRTEM image simulations.

Electronic Supplementary Material: Further HRTEM images and three HRTEM movies showing the layerby-layer sublimation of graphene, the wrapping of a segment of an MLE to form a BLE and the subsequent unwrapping, and the layer-by-layer removal process in a two-nested bilayer and a sandwiched monolayer graphene are available in the online version of this article at http://dx.doi.org/10.1007/s12274-010-1006-4 and are accessible free of charge. Movies may also be downloaded from http://alum.mit.edu/www/liju99/ Papers/10/Huang10/.

\section{References}

[1] Geim, A. K.; Novoselov, K. S. The rise of graphene. Nat. Mater. 2007, 6, 183-191.

[2] Novoselov, K. S. Jiang, Z.; Zhang, Y.; Morozov, S. V.; Stormer, H. L.; Zeitler, U.; Maan, J. C.; Boebinger, G. S.; Kim, P.; Geim, A. K. Room-temperature quantum Hall effect in graphene. Science 2007, 315, 1379-1379.

[3] Lee, C.; Wei, X. D.; Kysar, J. W.; Hone, J. Measurement of the elastic properties and intrinsic strength of monolayer graphene. Science 2008, 321, 385-388.

[4] Balandin, A. A.; Ghosh, S.; Bao, W. Z.; Calizo, I.; Teweldebrhan, D.; Miao, F.; Lau, C. N. Superior thermal conductivity of single-layer graphene. Nano Lett. 2008, 8 , 902-907.

[5] Girit, C. O.; Meyer, J. C.; Erni, R.; Rossell, M. D.; Kisielowski, C.; Yang L.; Park, C. -H.; Crommie, M. F.; Cohen, M. L.; Louie, S. G.; Zettl, A. Graphene at the edge: Stability and dynamics. Science 2009, 323, 1705-1708.

[6] Warner, J. H.; Rummeli, M. H.; Ge, L.; Gemming, T.; 
Montanari, B.; Harrison, N. M.; Buchner, B.; Briggs, G. A. D Structural transformations in graphene studied with high spatial and temporal resolution. Nat. Nanotechnol. 2009, 4, 500-504.

[7] Liu, Z.; Suenaga, K.; Harris, P. J. F.; Iijima, S. Open and closed edges of graphene layers. Phys. Rev. Lett. 2009, 102, 015501 .

[8] Jin, C.; Lan, H. P.; Peng, L. M.; Suenaga, K.; Iijima, S. Deriving carbon atomic chains from graphene. Phys. Rev. Lett. 2009, 102, 015501.

[9] Gass, M. H.; Bangert, U.; Bleloch, A. L.; Wang, P.; Nair, R. R.; Geim, A. K. Free-standing graphene at atomic resolution. Nat. Nanotechnol. 2008, 3,676-681.

[10] Jia, X. T.; Hofmann, M.; Meunier, V.; Sumpter, B. G.; Campos-Delgado, J.; Romo-Herrera, J. M.; Son, H.; Hsieh, Y. -P.; Reina, A.; Kong, J.; Terrones, M.; Dresselhaus M. S. Controlled formation of sharp zigzag and armchair edges in graphitic nanoribbons. Science 2009, 323, 1701-1705.

[11] Huang, J. Y.; Ding, F.; Yakobson, B. I.; Lu, P.; Qi, L.; Li, J. In situ observation of graphene sublimation and multi-layer edge reconstructions. Proc. Nat. Acad. Sci. USA 2009, 106, 10103-10108.

[12] Koskinen, P.; Malola, S.; Hakkinen, H. Self-passivating edge reconstructions of graphene. Phys. Rev. Lett. 2008, 101, 115502.

[13] Koskinen, P.; Malola S.; Hakkinen, H. Evidence for graphene edges beyond zigzag and armchair. Phys. Rev. B 2009, 80, 073401.

[14] Ohta, T.; Bostwick, A.; Seyller, T.; Horn, K.; Rotenberg, E. Controlling the electronic structure of bilayer graphene.
Science 2006, 313, 951-954.

[15] Huang, J. Y.; Chen, S.; Jo, S. H.; Wang, Z.; Han, D. X.; Chen, G.; Dresselhaus, M. S.; Ren, Z. F. Atomic-scale imaging of wall-by-wall breakdown and concurrent transport measurements in multiwall carbon nanotubes. Phys. Rev. Lett. 2005, 94, 236802.

[16] Iijima, S. Helical microtubules of graphitic carbon. Nature 1991, 354, 56-58.

[17] Huang, J. Y.; Ding, F.; Yakobson, B. I. Dislocation dynamics in multiwalled carbon nanotubes at high temperatures. Phys. Rev. Lett. 2008, 100, 035503.

[18] Feng, J.; Qi, L.; Huang, J. Y.; Li, J. Geometric and electronic structures of graphene bilayer edges. Phys. Rev. B 2009, 80, 165407.

[19] Egerton, R. F.; Li, P.; Malac, M. Radiation damage in the TEM and SEM. Micron 2003, 35, 399-409.

[20] Huang, J. Y. In situ observation of quasimelting of diamond and reversible graphite-diamond phase transformations. Nano Lett. 2007, 7, 2335-2340.

[21] Collins, P. C.; Arnold, M. S.; Avouris, P. Engineering carbon nanotubes and nanotube circuits using electrical breakdown. Science 2001, 292, 706-709.

[22] Jin, C.; Suenaga, K.; Iijima, S. Direct evidence for lip-lip interactions in multiwalled carbon nanotubes. Nano Res. 2008, 1, 434-439.

[23] Huang, J. Y.; Ding, F.; Yakobson, B. I. Vacancy-hole and vacancy-tube migration in multiwall carbon nanotubes. Phys. Rev. B, 2008, 78, 155436.

[24] Jin, C.; Suenaga, K.; Iijima, S. Vacancy migrations in carbon nanotubes. Nano Lett. 2008, 8, 1127-1130. 